\title{
Effect of magnetic field on the performance of a deep eutectic solvent-based redox flow battery from polarization perspective
}

\author{
Rong Cheng ${ }^{1,2}$, Qiang $\mathrm{Ma}^{1}$,Weiqi Zhang ${ }^{1}$, Huaneng $\mathrm{Su}^{1}$, and Qian $\mathrm{Xu}^{1, *}$ \\ ${ }^{1}$ Institute for Energy Research, Jiangsu University, 212013 Zhen jiang, China \\ ${ }^{2}$ School of Energy and Power Engineering, Jiangsu University, 212013 Zhen jiang, China
}

\begin{abstract}
In recent years, with the promotion of global renewable energy, the introduction of energy storage batteries has become more and more important. Redox flow battery has attracted more and more attention due to their environmental friendliness, safe and reliable operation. This work studies the effect of different magnetic field intensities and the duration of magnetic field addition on the mass transfer and electrochemical reaction process of iron-vanadium ions in deep eutectic solvent (DES). At the same time, the effect of magnetic field on the polarization performance of DES-based redox flow battery is also studied. The experimental results show that the iron and vanadium DES electrolytes increase their redox peak current density under the action of adding magnetic field. The test of the polarization performance of the DES-based redox flow battery shows that the addition of magnetic field can reduce the area specific resistance (ASR) of the battery and improve the polarization performance of the battery.
\end{abstract}

\section{Introduction}

In recent years, with the promotion of global renewable energy, from the perspective of power system stability, the introduction of energy storage batteries has become more and more important. Redox flow battery (RFB) has the advantages of environmental friendliness, flexible design, high energy efficiency, safe and reliable operation, fast startup speed, etc., and can solve the problems of discontinuity, instability and uncontrollability [1,2,3]. So as to accelerate the utilization of renewable energy, it has received more and more attention [4]. RFB has a long research history. Thaller of NASA proposed the principle of RFB "system" in 1974 [5]. Almost at the same time, Nozaki and others started the basic research and system development of $\mathrm{Fe} / \mathrm{Cr} \mathrm{RFB}$ at the National Institute of Advanced Industrial Science and Technology (AIST) in Japan [6]. Then in 1986, Professor Skylras Kazacos of the University of New South Wales in Australia invented the V/V RFB. The performance of the RFB system has been greatly improved, and the V-RFB is developing towards practicality [7]. The current aqueous redox flow battery has problems such as narrow electrochemical window $(<2 \mathrm{~V})$, which limits its further development to a certain extent. The use of non-aqueous electrolyte can overcome this shortcoming. Non-aqueous electrolyte can provide an extended stable potential window and expand the range of working voltage and working temperature. Therefore, RFB with non-aqueous electrolyte has the ability to provide high battery voltage and can provide higher energy density. This work uses a relatively lowcost ionic liquid analogue, namely deep eutectic solvent
(DES), to study the effect of different magnetic field intensities and the duration of magnetic field addition on the mass transfer and electrochemical reaction process of iron-vanadium ions in DES. At the same time, the influence of magnetic field on the polarization performance of deep eutectic solvent-based flow battery is also studied.

\section{Experimental}

\subsection{Preparation of electrolyte}

Choline chloride and cholesterol follow a 1:2 molar ratio of DES prepared by mixing. By heating a magnetic stirrer, heating to $120^{\circ} \mathrm{C}$ and stirring continuously for 20 min until a colorless and transparent liquid is formed, and then cooling the electrolyte at room temperature. After the DES electrolyte is ready, the redox active materials $\mathrm{FeCl} 3$ and $\mathrm{VCl} 3$ with a concentration of $0.1 \mathrm{M}$ are added to the DES respectively, and then a constanttemperature heating magnetic stirrer is used for continuous stirring at a heating temperature of $120^{\circ} \mathrm{C}$ for 2 hours. The two DES electrolytes are successfully prepared. In the heating process, to prevent volatilization, a layer of plastic wrap is attached to the mouth of the container.

\subsection{Electrochemical characterizations}

This experiment uses Chenhua CHI600 electrochemical workstation to test the cyclic voltammetry of the

\footnotetext{
* Corresponding author: xuqian@ujs.edu.cn
} 
electrolyte related experiments. The experiment uses a traditional three-electrode system for testing, in which the working electrode is a graphite electrode, the counter electrode is a platinum electrode, and the reference electrode is a saturated calomel electrode (SCE). Before the test, the electrolyte is purged with high-purity nitrogen for 20 minutes to remove the oxygen dissolved in the solution. Two DES electrolytes with different magnetic field intensities are scanned by cyclic voltammetry, and each experiment is repeated three times.

\subsection{Single cell performance test}

The internal structure of the battery is similar to the design of Leung et al. [8], and the design of the entire flow battery system is similar to that of Q. Xu et al. [9]. The assembled battery is composed of ion exchange membrane Nafion 115 membrane, graphite felt electrode with good conductivity, sealing gasket and graphite clamp. The parallel flow field is distributed on the contact surface of the graphite clamps on both sides. The bolts fixed on the surface of the fixture are used as collectors. The positive electrolyte is a DES electrolyte containing $0.1 \mathrm{M} \mathrm{FeCl} 2$, and the initial electrolyte of the negative electrolyte is a DES electrolyte containing 0.1 $\mathrm{M} \mathrm{VCl3.} \mathrm{The} \mathrm{electrolyte} \mathrm{is} \mathrm{stored} \mathrm{in} \mathrm{a} \mathrm{storage} \mathrm{tank}$ outside the battery, and the electrolyte is cyclically pumped to the corresponding reaction zone through a peristaltic pump during the operation of the battery. The loaded magnetic field under various working conditions is generated by WD-80 electromagnet generator. By adjusting the current value of the constant power supply from $0 \mathrm{~A}-4 \mathrm{~A}$, the corresponding uniform magnetic field intensity can be generated from $0 \mathrm{mT}-325 \mathrm{mT}$. In the charge-discharge cycle experiment, the state of charge (SOC) at the end of charging is set to $85 \%$, and it drops to about $15 \%$ at the end of discharge. The assembled full battery is placed in a magnetic field generator to test the polarization curves of the battery under different magnetic field intensities.

\section{Results and discussion}

\subsection{Electrochemical characteristics}

\subsubsection{Effect of magnetic field intensity}

The DES electrolyte containing $0.1 \mathrm{M} \mathrm{FeCl}_{3}$ and $0.1 \mathrm{M}$ $\mathrm{VCl}_{3}$ are placed in the magnetic field generator under different magnetic field intensities. The cyclic voltammetry curves are shown in Fig. 1. It can be seen from Fig. 1 (a) that at $0 \mathrm{mT}$, the peak current density of oxidation peak of iron DES electrolyte is 11.260 $\mathrm{mA} \cdot \mathrm{cm}-2$, and the peak current density of reduction peak is $-10.97 \mathrm{~mA} \cdot \mathrm{cm}-2$; when the magnetic field intensity is $325 \mathrm{mT}$, the redox peak current density of iron DES electrolyte is $12.640 \mathrm{~mA} \cdot \mathrm{cm}-2$ and $-12.36 \mathrm{~mA} \cdot \mathrm{cm}-2$, respectively. It can be seen from Fig. 1 (b) that the peak redox current density of vanadium DES electrolyte under the action of the added magnetic field, the increase reach $12.45 \%$ and $17.27 \%$ respectively. With the increase of the magnetic field intensity, the redox current density of the two DES electrolytes both increase. The movement of ions is accelerated, which makes electrochemical reactions easier.
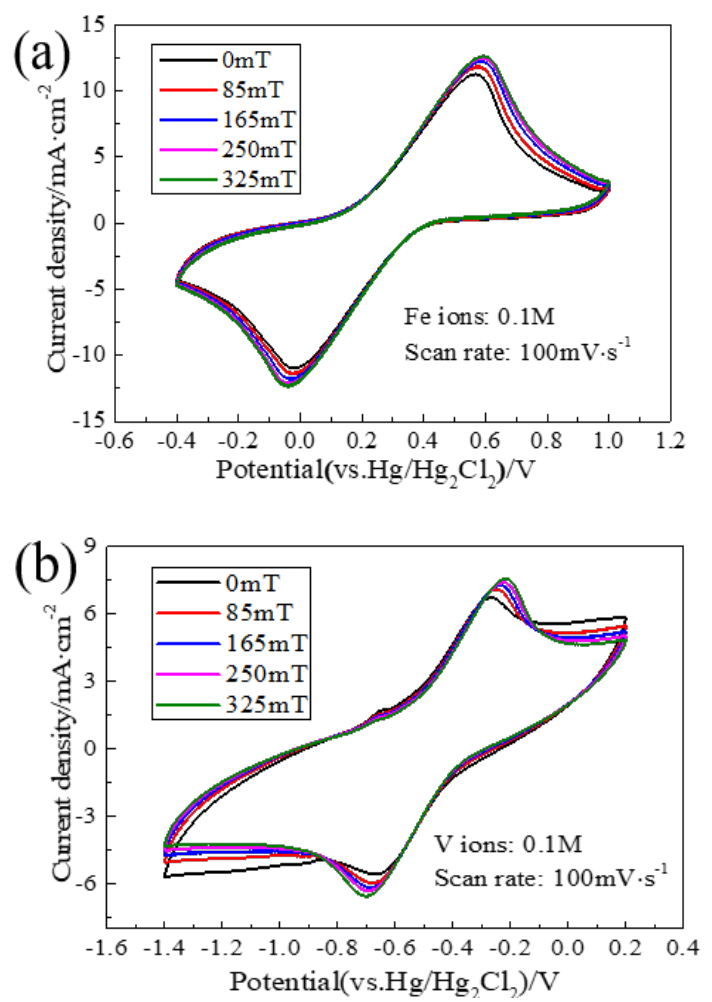

Fig. 1. CV curves of DES electrolytes adding different magnetic field intensities, scan rate of $100 \mathrm{mV} \cdot \mathrm{s}^{-1}$, (a) $0.1 \mathrm{M} \mathrm{Fe}$ ions; (b) $0.1 \mathrm{M} \mathrm{V}$ ions.

\subsubsection{Magnetic field timeliness analysis}

It can be seen from Fig. 2 that within 1 hour of removing the magnetic field from the $0.1 \mathrm{M} \mathrm{FeCl}_{3}$ DES electrolyte, the electrolyte redox peak current density increases. This indicates that the oxidation-reduction rate of the electrolyte at this stage is showing an increasing trend. After removing the magnetic field from the iron DES electrolyte for 1 hour, the redox peak current density of the electrolyte showed a downward trend. This indicates that the oxidation-reduction rate of the electrolyte at this stage shows a downward trend. It can be seen that the oxidation-reduction rate of $0.1 \mathrm{M} \mathrm{FeCl}_{3}$ DES electrolyte reaches its maximum value before and after removing the magnetic field for 1 hour, and then it shows a downward trend. In addition, analyzing the data in Fig. 2 knows that the peak potential difference $(\Delta E)$ first increases and then decreases with the increase in the time of separation from the magnetic field. At $0 \mathrm{~h} \Delta E$ is $0.670 \mathrm{~V}$, at $1 \mathrm{~h} \Delta E$ increases to $0.738 \mathrm{~V}$, and then shows a downward trend. The change in the potential difference between the peak currents indicates that the reversibility of the redox reaction first weakens and then increases during a period of time when the magnetic field intensity is removed. It can be seen that scientific control of the 
time for the iron DES electrolyte to leave the magnetic field intensity can improve the characteristics of electrochemically active materials.

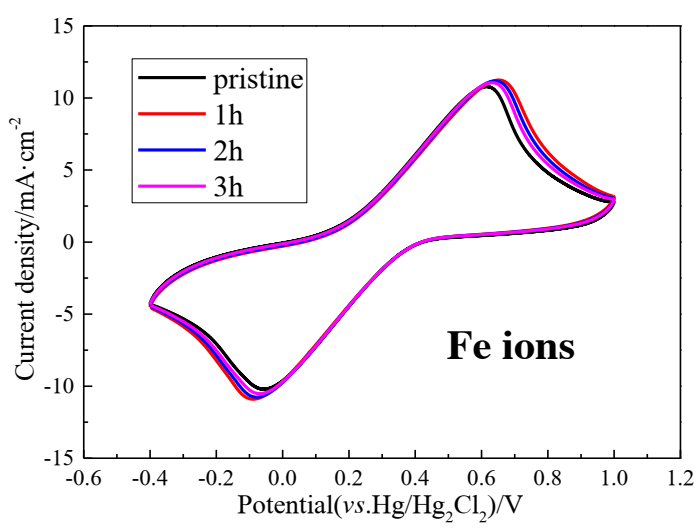

Fig. 2. $\mathrm{CV}$ curves of $0.1 \mathrm{M} \mathrm{FeCl} \mathrm{FeS}_{3}$ DES electrolyte removing magnetic field at different times, scan rate is $100 \mathrm{mV} \cdot \mathrm{s}^{-1}$.

It can be seen from Fig. 3 that after the $0.1 \mathrm{M} \mathrm{VCl}_{3}$ electrolyte removes the magnetic field, the electrolyte redox peak current density decreases with time. This indicates that the oxidation-reduction rate of the electrolyte shows a decreasing trend. The peak potential difference $(\Delta E)$ increases first and then stabilizes with the increase of the time of leaving the magnetic field. $\Delta E$ is $0.337 \mathrm{~V}$ at $0 \mathrm{~h}$, and $\Delta E$ increased to $0.606 \mathrm{~V}$ at $3 \mathrm{~h}$, and then is stabilized. The increase in the potential difference between the peak currents indicates that the reversibility of the oxidation-reduction reaction of the vanadium electrolyte is poor compared with the initial data during a period of time when the magnetic field intensity is removed. It can be seen that the electrochemical characteristics of the vanadium DES electrolyte become worse when it is separated from the magnetic field intensity, namely it is immediately affected by the magnetic field effect, and the positive effect it receives is disappeared after the magnetic field disappears.

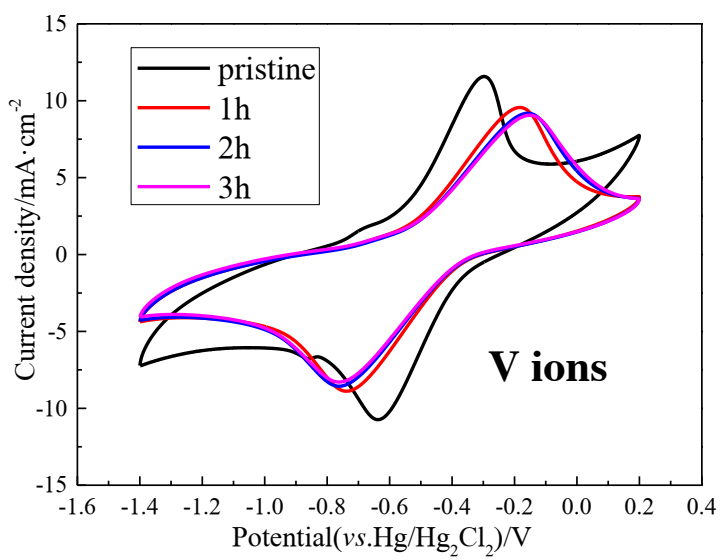

Fig. 3. $\mathrm{CV}$ curves of $0.1 \mathrm{M} \mathrm{VCl} \mathrm{Vl}_{3}$ DES electrolyte removing magnetic field at different times, scan rate is $100 \mathrm{mV} \cdot \mathrm{s}^{-1}$.

\subsection{Single cell performance}

In order to study the influence of different magnetic field intensities on battery power density, polarization curves tests are performed on DES-based redox flow battery with different magnetic field intensities added. The battery discharge polarization curves are used to study the effect of different magnetic field intensities on the polarization performance of DES-based redox flow battery. Fig. 4 exhibits the area specific resistance (ASR) of the battery with different magnetic field intensities. The result shows that when the magnetic field intensity is $325 \mathrm{mT}$, the ASR of the battery can be reduced by $16.65 \%$ compared with the case without the magnetic field. It can be seen from Fig. 5 that compared with the original data without adding the magnetic field, the battery with adding the magnetic field shows better discharge performance. Under the $325 \mathrm{mT}$ magnetic field, the battery energy efficiency has increased from $53.20 \%$ to $56.37 \%$, and the limiting current density and power density of the battery have also been significantly improved. They increase from $11.39 \mathrm{~mA} \cdot \mathrm{cm}^{-2}$ and $2.81 \mathrm{~mW} \cdot \mathrm{cm}^{-2}$ to $14.97 \mathrm{~mA} \cdot \mathrm{cm}^{-2}$ and $3.48 \mathrm{~mW} \cdot \mathrm{cm}^{-2}$, respectively. This shows that within a certain range, as the intensity of the loaded magnetic field increases, the power density of the battery also increases. It further shows that adding magnetic field optimizes the polarization performance of the DES-based redox flow battery.

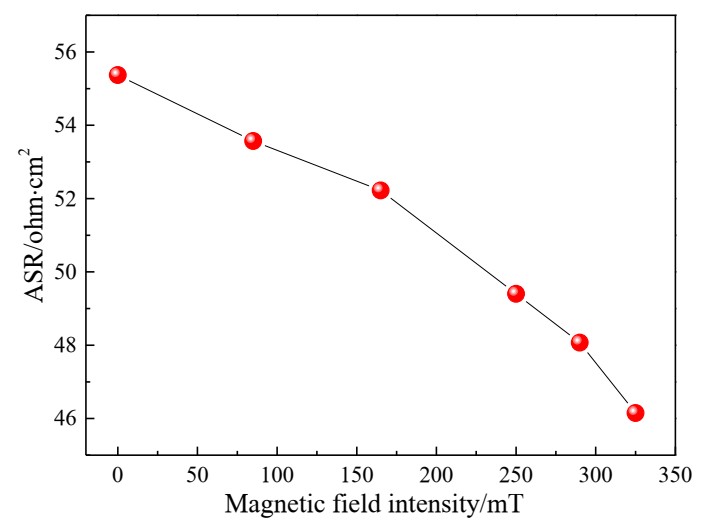

Fig. 4. ASR of the battery with DES-based electrolyte under different magnetic field intensities.

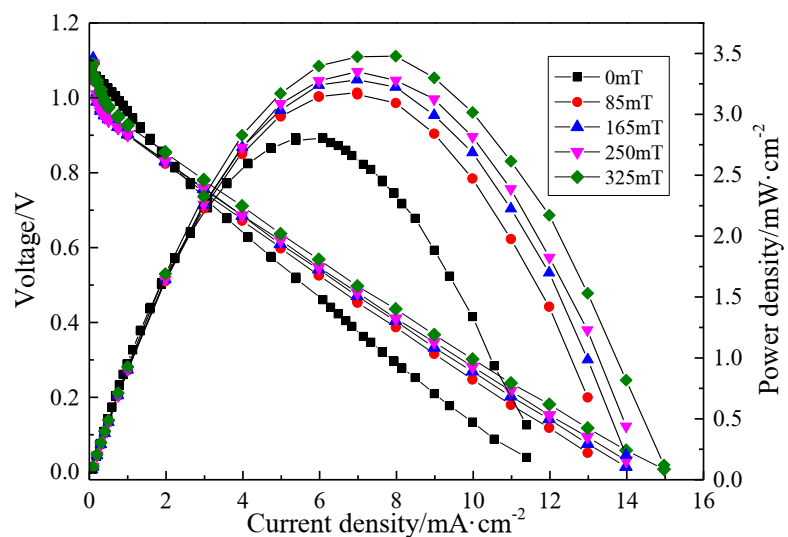

Fig. 5. Power density curves and polarization curves of battery under different magnetic field intensities. 


\section{Conclusion}

This work studies the effect of magnetic field intensities and the duration of the added magnetic field on the mass transfer and electrochemical reaction process of ironvanadium ions in DES. Also, the effect of the external magnetic field on the polarization performance of the DES-based redox flow battery is studied. By studying the electrochemical characteristics of iron-vanadium DES electrolyte with different magnetic field intensities, it is concluded that as the magnetic field intensity increases, the redox peak current density of the ironvanadium DES electrolyte both increase, which indicates that the rate of oxidation-reduction reaction of the electrolyte increases due to the enhancement of the magnetic field intensity, the movement of ions is accelerated, which the electrochemical reaction is easier to proceed. Through the study of the timeliness of the magnetic field effect of the iron-vanadium DES electrolyte, the experimental results show that the electrochemical characteristics of the iron DES electrolyte increase within one hour after the magnetic field is withdrawn, and the electrochemical characteristics of the vanadium DES electrolyte begin when the magnetic field is withdrawn. It indicates that the timeliness of the magnetic field effect of the iron DES electrolyte is better than that of the vanadium DES electrolyte. The polarization performance test of DESbased redox flow battery with external magnetic field shows that adding magnetic field can reduce the ASR of DES-based redox flow battery and improve the polarization performance of the battery. It is worth mentioning that when magnetic field of $325 \mathrm{mT}$ is added, the ASR of the DES-based redox flow battery is reduced by $16.65 \%$; The limiting current density and maximum power density are increased by $31.43 \%$ and $23.84 \%$, respectively. The underlying mechanism of adding magnetic field to optimize the polarization performance of DES electrolyte and DES-based redox flow battery needs further study.

\section{Acknowledgement}

The work described in this paper was fully supported by Grants from NSFC, China (No. 51676092), Six-Talent-Peaks Project in Jiangsu Province (2016-XNY-015), High-Tech Research Key Laboratory of Zhenjiang City (No. SS2018002), and a Project Funded by the Priority Academic Program Development of Jiangsu Higher Education Institutions (PAPD), China.

\section{References}

1. S. Kim, M. Vijayakumar, W. Wang, et al., Phys. Chem. Chem. Phys. 13, 18186 (2011)

2. J.A. Turner, Science 285, 687-689 (1999)

3. Z.G. Yang, J.L. Zhang, M.C. Kintner-Meyer, et al., Chem. Rev. 111, 3577-3613 (2011)

4. F. Díaz-González, A. Sumper, O. Gomis-Bellmunt, et al., Renew. Sustain. Energy Rev. 16, 2154-2171 (2012)
5. L.H. Thaller, Proc. 9th IECEC, 924-928(1974)

6. K. Nozaki, H. Kaneko, T. Ozawa, O. Hamamoto, Electrochemical and Electro-Heat Work Shop. CH75-3, IEEJ (1975)

7. E. Sum, M. Rychcik and M. Skyllas-Kazacos, J. Power Sources 16, 85-95(1985)

8. P.K. Leung, M.R. Mohamed, A.A. Shah, Q. Xu, J. Power Sources 274, 651-658(2015)

9. Q. Xu, L.Y. Qin, Y.N. Ji, P.K. Leung, H.N. Su, Electrochim. Acta 293, 426-431(2019) 